\title{
FIELD CONDITIONS AT THE MARICOPA AGRICULTURAL CENTER, PIMAL COUNTY, ARIZONA, SEPTEMBER 28, 1989
}

By Sandra J. Owen-Joyce

U.S. GEOLOGICAL SURVEY

Open-File Report 89-590

Prepared in cooperation with the

U.S. BUREAU OF RECLAMATION 
DEPARTMENT OF THE INTERIOR

MANUEL LUJAN, JR., Secretary

U.S. GEOLOGICAL SURVEY

Dallas L. Peck, Director

For additional information write to:

District Chief

U.S. Geological Survey

Federal Building, Box FB-44

300 West Congress Street

Tucson, Arizona 85701-1393
Copies of this report can be purchased from:

U.S. Geological Survey Books and Open-File Reports Section Federal Center, Bldg. 810

Box 25425

Denver, Colorado 80225 


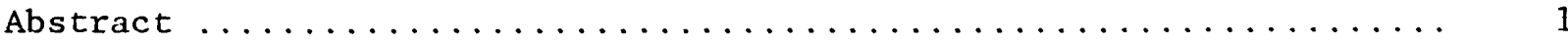

Introduction $\ldots \ldots \ldots \ldots \ldots \ldots \ldots \ldots \ldots \ldots \ldots \ldots \ldots \ldots \ldots \ldots$

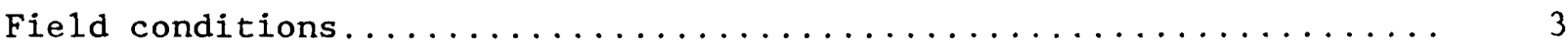

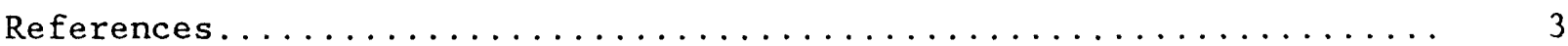

FIGURES

Figure 1. Location of the Maricopa Agricultural Center, Pinal

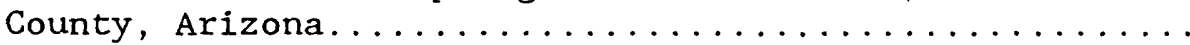

2. Field boundaries for the Maricopa Agricultural Center

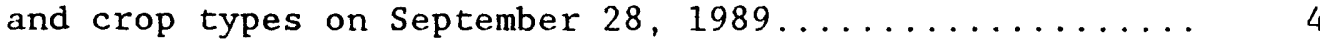

3. Location and view direction of photographs taken to document field conditions on the demonstration farm, Maricopa Agricultural Center, September 28, 1989.......

TABLES

Table 1. Ground-truth data for fields on the demonstration farm, Maricopa Agricultural Center, on September 28, 1989.....

2. Photographs (35-millimeter slides) taken of fields on the demonstration farm, Maricopa Agricultural Center, and of scientists and equipment on September $28,1989 \ldots$. 


\section{CONVERSION FACTORS}

For readers who prefer to use inch-pound units rather than metric (International System) units, the conversion factors for the terms used in this report are listed below:

Multiply inch-pound unit
millimeter (mm)
meter (m)
kilometer $(\mathrm{km})$

By

0.03937

3.2818

0.6214
To obtain metric unit

inch (in.)

foot ( $f t)$

mile (mi)

Sea level: In this report "sea level" refers to the National Geodetic Vertical Datum of 1929 (NGVD of 1929) - A geodetic datum derived from a general adjustment of the first-order level nets of both the United States and Canada, formerly called "Sea Level Datum of 1929." 
FIELD CONDITIONS AT THE MARICOPA AGRICULTURAL CENTER, PINAL COUNTY, ARIZONA, SEPTEMBER 28, 1989

By

Sandra J. Owen-Joyce

\begin{abstract}
Field conditions were documented during the Landsat and SPOT satellite overpasses of the Maricopa Agricultural Center, Pinal County, Arizona, on September 28, 1989. Crop types were mapped and photographed for each demonstration farm field, and irrigation, cultivation, and orientation of rows are described. Field and photographic descriptions are presented in tabular and graphic form.
\end{abstract}

INTRODUCTION

The Remote Sensing Technology Transfer Experiment is being run at the University of Arizona Maricopa Agricultural Center (MAC) ( $f i g .1$ ) by the University of Arizona to demonstrate the feasibility of remote-sensing techniques as an agricultural management tool. The purpose of the experiment is to investigate and implement potential commercial applications of this technology to agriculture with a long-term goal of providing the farmer with a "real time" management tool for (1) detection and early warning indicators of pest, water, and nutrient stress problems; (2) yield forecasting; and (3) efficient application of water, nutrients, and biologic pest-control substances.

The U.S. Geological Survey, in cooperation with the U.S. Bureau of Reclamation, is conducting research to evaluate the spatial and temporal variability of point data collected at weather stations for use with satellite-image data in estimating local evapotranspiration. The data collection at the weather stations is being done under contract with the University of Arizona. This work is being done at MAC during the Remote Sensing Technology Transfer Experiment under an umbrella agreement whereby scientists that work independently can cooperatively share the data collected. This report documents the field conditions at the time of the Landsat and SPOT overpasses on September 28, 1989.

Field conditions have been documented previously at MAC to correspond with the acquisition of satellite imagery. Data on crop types are available for June 1988 (Owen-Joyce, 1988), April 1989 (Owen-Joyce, 1989a), and June 1989 (Owen-Joyce, 1989b). 

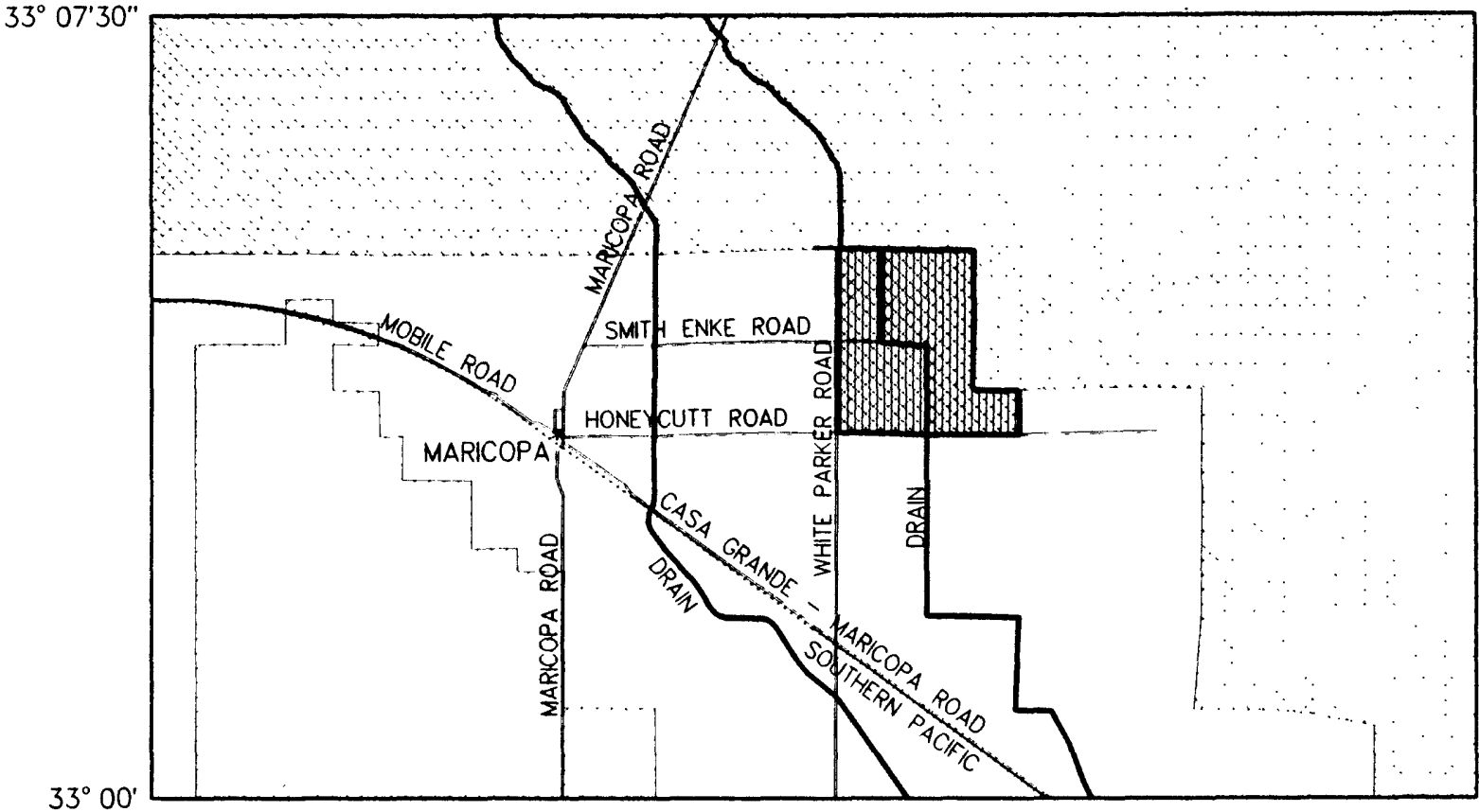

$$
\begin{array}{lllllllll}
0 & & 1 & & 2 & & 3 & 4 & 5 \\
1 & 1 & 1 & 1^{1} & 1 & 1 & 1 & 1 & 1 \\
0 & 1 & 2 & 3 & 4 & 5 & \text { MLOLSES }
\end{array}
$$

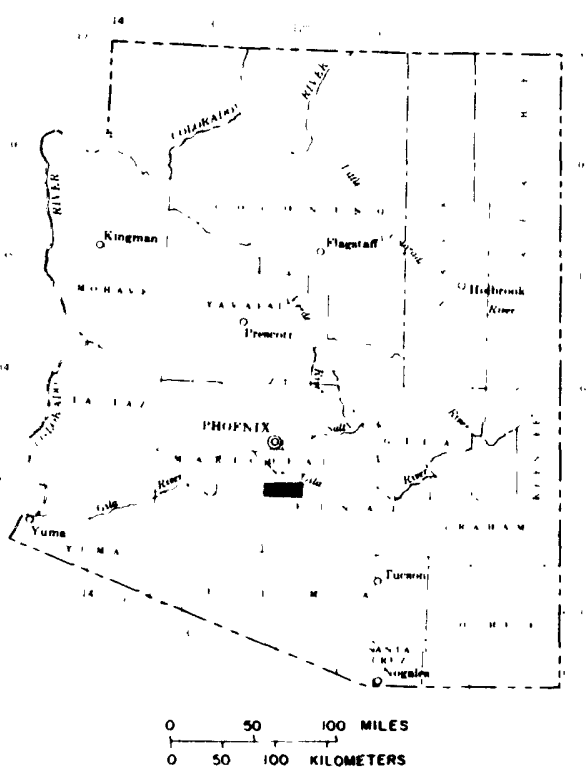

EXPLANATION

INDEX MAP SHOWING STLDY

AREA (SHADED)

Figure 1.--Location of the Maricopa Agricultural Center, Pinal County, Arizona. 


\section{FIELD CONDITIONS}

Ground-truth data were collected to coincide with the Landsat and SPOT satellite overpasses on September 28, 1989. The data include crop type for each field on the demonstration farm, a description of the crop and field conditions, and photographs (35-millimeter slides) of the fields. Photographs were taken on September 28 to document field conditions and to provide ground truth for interpretation of the satellite data. Photographs were taken also on September 28 of participating scientists and their equipment during data collection.

Crop types and descriptions of the fields are presented in table 1. Field boundaries and the crop type in each demonstration farm field are shown in figure 2. The generalized location from which each photograph was taken, the direction of view, and a brief description of conditions that the photograph documents are listed in table 2 . The location from which photographs documenting field conditions were taken are shown in figure 3; where possible, these photographs were taken viewing $180^{\circ}$ away from the sun. Selected slides were duplicated and are available at the U.S. Water Conservation Laboratory, Phoenix, Arizona; these slides are footnoted in table 2 .

Conditions were also noted in the fields outside the farm but immediately adjacent to boundary roads of the demonstration farm. Cotton is planted east of field 11, north of fields 25, 26, and 39, northwest of field 39, west of fields 37-39, and southeast of field 27. North of fields 19-22 is natural desert vegetation. Fields east of field 19 and north of field 23 are fallow. Southwest of field 27 is natural desert vegetation and houses. South of the west half of field 27 , cotton is planted. South of the east half of field 27, cotton is planted in the east half and the west half is fallow. West of fields 27 to 3201 , the land is planted with mature pecan trees. West of fields 33-36, the fallow land has some weeds.

\section{REFERENCES}

Owen-Joyce, S.J., 1988, Field conditions at the Maricopa Agricultural Center, Maricopa County, Arizona, June 13, 1988: U.S. Geological Survey Open-File Report 88-708, 5 p.

Owen-Joyce, S.J., 1989a, Field conditions at the Maricopa Agricultural Center, Pinal County, Arizona, April 9, 1989: U.S. Geological Survey Open-File Report 89-377, 14 p.

Owen-Joyce, S.J., 1989b, Field conditions at the Maricopa Agricultural Center, Pinal County, Arizona, June 16, 1989: U.S. Geological Survey Open-File Report 89-392, 11 p. 


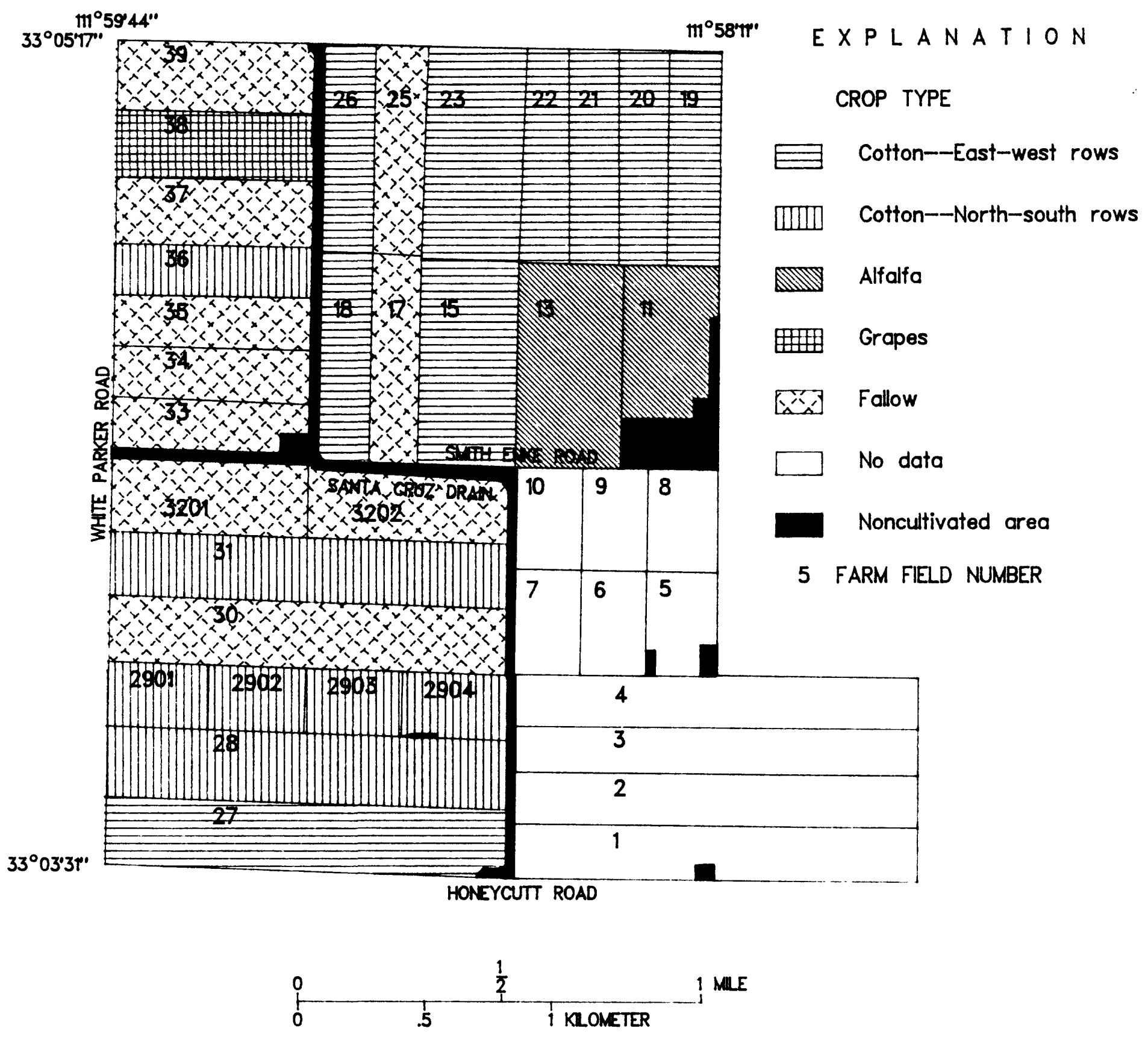

Figure 2.--Field boundaries for the Maricopa Agricultural Center and crop types on September 28, 1989. 


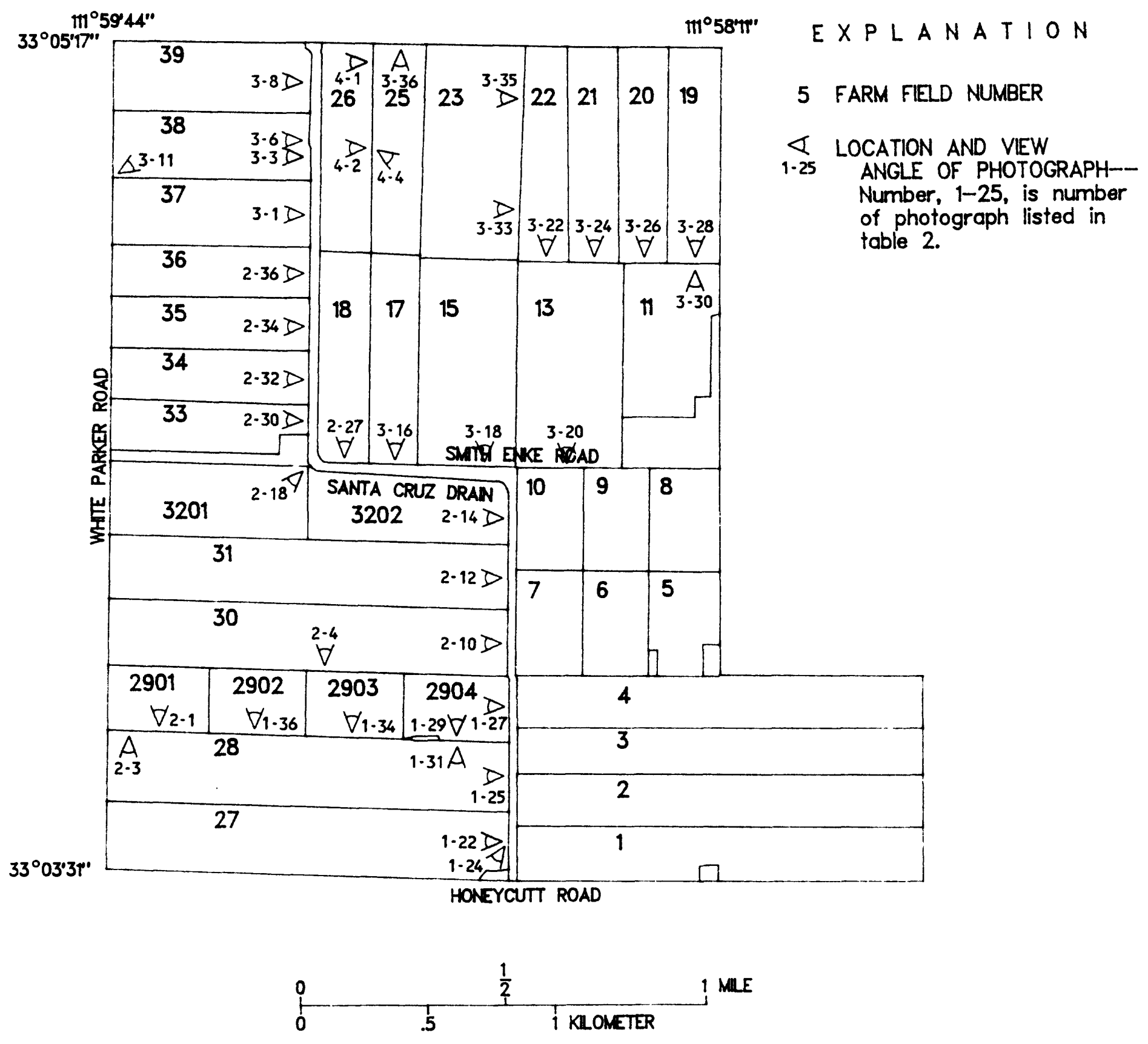

Figure 3.--Location and view direction of photographs taken to document field conditions on the demonstration farm, Maricopa Agricultural Center, September 28, 1989. 


$\begin{array}{ccc}\begin{array}{c}\text { Maricopa Agricultural } \\ \text { Center }\end{array} & \text { Crop } & \text { Description } \\ \text { Field Number } & & \end{array}$

Field Number 1

11

13

15

17

18

19

20

21

22

23

25

26

27

28

2901

2902

2903

2904

$$
30
$$

$$
31
$$

3201

3202

$$
33
$$

$$
34
$$$$
35
$$

$$
36
$$$$
37
$$$$
38
$$

39
Alfalfa

Alfalfa

Cotton

Fallow

Cotton

Cotton

Cotton

Cotton

Cotton

Cotton

Fallow

Cotton

Cotton

Cotton

Cotton

Cotton

Cotton

Cotton

Fallow

Cotton

Fallow

Fallow

Fallow

Fallow

Fallow

Cotton

Fallow

Grapes

Fallow
Soil damp. Buildings in south half.

Soil damp.

East-west rows. Dry.

Dry.

East-west rows. Dry.

East-west rows. Dry.

East-west rows. Dry.

East-west rows. Dry.

East-west rows. Dry.

East-west rows. Dry.

Dry.

East-west rows. Dry. North $45 \mathrm{~m}$ fallow.

East-west rows. Dry. Drip irrigated field. East end has carbon dioxide rings set up.

North-south rows, Dry.

North-south rows. Dry.

North-south rows. Dry.

North-south rows. Dry.

North-south rows. Dry.

Plowed, clods. Dry.

North-south rows. Dry.

Plowed, rounded clods, some stubble. Dry.

Same as field 3201.

Plowed, some stubble, clods. Dry.

Plowed, clods, some stubble. Dry.

Plowed, clods, some stubble. Dry.

North-south rows. Dry.

Plowed, clods, some stubble. Dry.

North-south rows. Not cultivated. Dry. Many weeds around plants and between rows. Any grapes on vines are dried.

Plowed, clods, some stubble. Dry.

\footnotetext{
${ }^{1}$ Fields plotted on figure 2.
} 
Table 2. -- Photographs (35-millimeter slides) taken of fields on the demonstration farm. Maricopa Agricultural Center, and of scientists and equipment on September 28,1989

[Location, View direction, and Description: SE, Southeast; SW, Southwest; NE, Northeast; NW, Northwest; ESE, East southeast; ENE, East northeast; NNW, North northwest; WNW, West northwest. Description: MAC, Maricopa Agricultural Center]

\begin{tabular}{|c|c|c|c|c|c|c|}
\hline Date & Time & Slide & $\begin{array}{l}\text { Field } \\
\text { number }\end{array}$ & Location & $\begin{array}{c}\text { View } \\
\text { direction }\end{array}$ & Description \\
\hline $9 / 20 / 89$ & 0930 & $1-1$ & 20 & Middle & NNW & $\begin{array}{l}\text { Weather station in cotton field. Bruce } \\
\text { Russell and James Brown. }\end{array}$ \\
\hline$"$ & 0930 & $1-2$ & 20 & Middle & NNW & Weather station. \\
\hline$"$ & 0937 & $1-3$ & 20 & Middle & Close up & Cotton. \\
\hline$"$ & -- & $1-4$ & 20 & Middle & Close up & Cotton. \\
\hline$"$ & -- & $1-5$ & 20 & Middle & Close up & Cotton. \\
\hline$"$ & 0948 & $1-6$ & 20 & West side & East & Weather station in cotton field. \\
\hline$"$ & 1004 & $1-7$ & -- & West side & East & $\begin{array}{l}\text { Weather station in cotton field with } \\
\text { east-west rows. Murphy Farm. Bruce } \\
\text { Russell and James Brown. }\end{array}$ \\
\hline " & -- & $1-8$ & -- & Middle & West & Same as $1-7$ \\
\hline 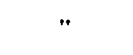 & -- & $1-9$ & -- & Middle & West & Same as $1-7$ \\
\hline$"$ & 1010 & $1-10$ & -- & Middle & SW & $\begin{array}{l}\text { Infrared sensor on weather station. } \\
\text { Murphy Farm. James Brown. }\end{array}$ \\
\hline$"$ & -- & $1-11$ & -- & Middle & WNW & Cotton. Murphy Farm. \\
\hline$"$ & 1015 & $1-12$ & -- & West side & East & $\begin{array}{l}\text { Weather station in cotton field. Murphy } \\
\text { Farm. }\end{array}$ \\
\hline$"$ & -- & $1-13$ & -- & Middle & South & $\begin{array}{l}\text { Weather station in cotton field with } \\
\text { north-south rows. Smith Farm. }\end{array}$ \\
\hline 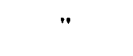 & 1031 & $1-14$ & -- & $"$ & West & Same as $1-13$ \\
\hline$"$ & 1031 & $1-15$ & -- & $"$ & West & Same as $1-13$ \\
\hline$"$ & 1039 & $1-16$ & -- & $"$ & NW & $\begin{array}{l}\text { Cotton plant and weather station. Smith } \\
\text { Farm. }\end{array}$ \\
\hline$"$ & 1039 & $1-17$ & -- & $"$ & NE & Cotton plant. Smith Farm. \\
\hline$"$ & 1043 & $1-18$ & -- & $"$ & Close up & Cotton flower. Smith Farm. \\
\hline$"$ & 1043 & $1-19$ & -- & $"$ & Close up & Cotton flower. Smith Farm. \\
\hline$"$ & -- & $1-20$ & -- & West side & East & $\begin{array}{l}\text { Weather station in cotton field with } \\
\text { east-west rows. Ak-Chin Farm. }\end{array}$ \\
\hline$"$ & -- & $1-21$ & -- & West side & East & Same as $1-20$ \\
\hline $9 / 28 / 89$ & 0924 & $1_{1-22}$ & 27 & East side & West & $\begin{array}{l}\text { Cotton. Dry. Crop duster plane-no } \\
\text { spraying. }\end{array}$ \\
\hline
\end{tabular}


Table 2. - Photographs (35-millimeter slides) taken of fields on the demonstration farm, Maricopa Asricultural Center, and of scientists and equipment on September 28, 1989--Continued

\begin{tabular}{|c|c|c|c|c|c|c|}
\hline Date & Time & Slide & $\begin{array}{l}\text { Field } \\
\text { number }\end{array}$ & Location & $\begin{array}{c}\text { View } \\
\text { direction }\end{array}$ & Description \\
\hline $9 / 28 / 89$ & 0924 & $1_{1-23}$ & 27 & East side & West & Same as $1-22$, closer view. \\
\hline 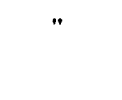 & 0924 & $1-24$ & 27 & $"$ & SW & $\begin{array}{l}\text { Cotton, carbon dioxide rings in bench }{ }^{2} 2 \\
\text { at east end of field. }\end{array}$ \\
\hline$"$ & 0928 & $11-25$ & 28 & $"$ & West & Cotton. Dry. \\
\hline$"$ & 9028 & $1_{1-26}$ & 28 & $"$ & West & Same as $1-25$, closer view. \\
\hline$"$ & 0931 & $1_{1-27}$ & 2904 & $"$ & West & Cotton. Dry. \\
\hline$"$ & 0931 & $1_{1-28}$ & 2904 & $"$ & West & Same as $1-27$, closer view. \\
\hline$"$ & 0933 & $1_{1-29}$ & 2904 & South side & North & Cotton. Dry. \\
\hline$"$ & 0933 & $1_{1-30}$ & 2904 & $"$ & North & Same as $1-29$, closer view. \\
\hline$"$ & 0935 & $11-31$ & 28 & North side & South & Cotton. Dry. \\
\hline$"$ & 0935 & $1_{1-32}$ & 28 & $"$ & South & Same as $1-31$, closer view. \\
\hline$"$ & 0938 & $1-33$ & 2904 & SE corner & NW & Well. \\
\hline$"$ & 0942 & $1_{1-34}$ & 2903 & South side & North & Cotton. Dry. \\
\hline$"$ & 0942 & $11-35$ & 2903 & $"$ & North & Same as $1-34$, closer view. \\
\hline$"$ & 0945 & $\mathbf{1}_{1-36}$ & 2902 & $"$ & North & Cotton. Dry. \\
\hline$"$ & 0945 & $1_{1-37}$ & 2902 & $"$ & North & Same as 1-36, closer view. \\
\hline$"$ & 0952 & $1_{2-1}$ & 2901 & $"$ & North & Cotton. Dry. \\
\hline$"$ & 0952 & $1_{2-2}$ & 2901 & $"$ & North & Same as $2-1$, closer view. \\
\hline$"$ & 0954 & $1_{2-3}$ & 28 & North side & South & $\begin{array}{l}\text { Cotton, alternating groups of rows of } \\
\text { different heights at west end. }\end{array}$ \\
\hline$"$ & 1000 & $2-4$ & 30 & South side & North & $\begin{array}{l}\text { Weather station in fallow field, plowed, } \\
\text { clods, some stubble. }\end{array}$ \\
\hline$"$ & 1000 & $2-5$ & 30 & South side & North & Same as $2-3$, closer view. \\
\hline$"$ & 1000 & $2-6$ & 30 & $"$ & $\mathrm{NE}$ & Paul Pinter walking sensors. \\
\hline$"$ & 1002 & $1_{2-7}$ & 30 & $"$ & North & Same as $2-6$ \\
\hline$"$ & 1004 & $1_{2-8}$ & 30 & $"$ & NW & Same as $2-6$ \\
\hline$"$ & 1006 & $2-9$ & 30 & SE corner & NW & Well. \\
\hline$"$ & 1008 & $1_{2-10}$ & 30 & East side & West & Fallow, plowed, clods. \\
\hline$"$ & 1008 & $12-11$ & 30 & $"$ & West & Same as $2-10$, closer view. \\
\hline$"$ & 1012 & $1_{2-12}$ & 31 & $"$ & West & Cotton. Dry. \\
\hline$"$ & 1012 & $1_{2-13}$ & 31 & $"$ & West & Same as 2-12, closer view. \\
\hline
\end{tabular}


Table 2. --Photographs (35-millimeter slides) taken of fields on the demonstration farm, Maricopa Agricultural Center, and of scientists and equipment on September 28, 1989--Cont inued

\begin{tabular}{|c|c|c|c|c|c|c|c|}
\hline Date & Time & Slide & $\begin{array}{l}\text { Field } \\
\text { number }\end{array}$ & Loc & tion & $\begin{array}{c}\text { View } \\
\text { direction }\end{array}$ & Description \\
\hline $9 / 28 / 89$ & 1015 & $1_{2-14}$ & 3202 & East & side & West & $\begin{array}{l}\text { Fallow, plowed, rounded clods, wheat } \\
\text { stubble. }\end{array}$ \\
\hline$"$ & 1015 & $1_{2-15}$ & 3202 & $"$ & $"$ & West & Same as $2-14$, closer view. \\
\hline$"$ & 1018 & $2-16$ & 15 & Soutl & side & North & People working. \\
\hline$"$ & 1018 & $1_{2-17}$ & 15 & $"$ & $"$ & NW & $\begin{array}{l}\text { Cessna }{ }^{3} \text { and people working. Santa Cruz } \\
\text { drain in foreground. }\end{array}$ \\
\hline$"$ & 1023 & $1_{2-18}$ & 3201 & NE cc & rner & SW & Fallow, same as field 3202 \\
\hline$"$ & 1023 & $1_{2-19}$ & 3201 & West & side & East & Same as $2-18$, closer view. \\
\hline$"$ & 1027 & $1_{2-20}$ & 17 & West & side & ENE & $\begin{array}{l}\text { Ron Seay and Susan Moran programing } \\
\text { sensors. }\end{array}$ \\
\hline$"$ & 1027 & $1_{2-21}$ & 18 & East & side & -- & Cessna over field 18 \\
\hline$"$ & 1028 & $2-22$ & 17 & West & side & -- & Cessna over field 17 . \\
\hline$"$ & 1030 & $12-23$ & 17 & $"$ & $"$ & East & Ron Seay and Susan Moran walking sensors. \\
\hline$"$ & 1032 & $1_{2-24}$ & 17 & $"$ & $"$ & East & $\begin{array}{l}\text { Ron Seay and Susan Moran walking sensors } \\
\text { in field } 17 \text { with Cessna and people } \\
\text { working near plane parking area. }\end{array}$ \\
\hline$"$ & 1033 & $1_{2-25}$ & 17 & $"$ & $"$ & -- & Sky condition, clear, hot, and breezy. \\
\hline$"$ & 1037 & $1_{2-26}$ & 17 & West & side & North & $\begin{array}{c}\text { Susan Moran, Ian Perry, and Karen Scott } \\
\text { calibrating sensors over white plate. }\end{array}$ \\
\hline$"$ & 1037 & $1_{2-27}$ & 18 & Soutr & side & North & Cotton, open areas in field. Dry. \\
\hline$"$ & 1037 & $1_{2-28}$ & 18 & $"$ & $"$ & North & Same as $2-27$, closer view. \\
\hline$"$ & 1040 & $2-29$ & 33 & SE cc & rner & NW & Well. \\
\hline$"$ & 1042 & $1_{2-30}$ & 33 & East & side & West & $\begin{array}{l}\text { Fallow, plowed, clods, some stubble. } \\
\text { Dry. }\end{array}$ \\
\hline$"$ & 1042 & $1_{2-31}$ & 33 & $"$ & $"$ & West & Same as $2-30$, closer view. \\
\hline$"$ & 1044 & $1_{2-32}$ & 34 & $"$ & $"$ & West & $\begin{array}{l}\text { Fallow, plowed, clods, some stubble. } \\
\text { Dry. }\end{array}$ \\
\hline$"$ & 1044 & $1_{2-33}$ & 34 & $"$ & $"$ & West & Same as $2-32$, closer view. \\
\hline$"$ & 1050 & $12-34$ & 35 & $"$ & $"$ & West & $\begin{array}{l}\text { Fallow, plowed, clods, some stubble. } \\
\text { Dry. }\end{array}$ \\
\hline$"$ & 1050 & $\mathbf{1}_{2-35}$ & 35 & $"$ & $"$ & West & Same as $2-34$, closer view. \\
\hline$"$ & 1056 & $1_{2-36}$ & 36 & $"$ & $"$ & West & Cotton. Dry. \\
\hline " & 1056 & $1_{2-37}$ & 36 & $"$ & $"$ & West & Same as 2-36, closer view. \\
\hline
\end{tabular}


Table 2. - Photographs (35-millimeter slides) taken of fields on the demonstration farm, Maricopa Agricultural Center, and of scientists and equipment on September 28, 1989--Continued

\begin{tabular}{|c|c|c|c|c|c|c|c|}
\hline Date & Time & Slide & $\begin{array}{l}\text { Field } \\
\text { number }\end{array}$ & Locat & tion & $\begin{array}{l}\text { View } \\
\text { direction }\end{array}$ & Description \\
\hline $9 / 28 / 89$ & 1101 & $1_{3-1}$ & 37 & East & side & West & $\begin{array}{l}\text { Fallow, plowed, clods, some stubble. } \\
\text { Dry. }\end{array}$ \\
\hline$"$ & 1101 & $1_{3-2}$ & 37 & $"$ & $"$ & West & Same as $3-1$, closer view. \\
\hline$"$ & 1105 & $1_{3-3}$ & 38 & $"$ & $"$ & West & Grapes with weeds. Dry. \\
\hline$"$ & 1105 & $1_{3-4}$ & 38 & $"$ & $"$ & West & Same as $3-3$, closer view. \\
\hline$"$ & 1105 & $3-5$ & 38 & $"$ & $"$ & NW & Well. \\
\hline$"$ & 1106 & $1_{3-6}$ & 38 & $"$ & $"$ & West & $\begin{array}{l}\text { Open area in center of grapes with } \\
\text { weeds. }\end{array}$ \\
\hline$"$ & 1108 & $1_{3-7}$ & 38 & $\mathrm{NE} \operatorname{col}$ & rner & SW & Grapes. \\
\hline$"$ & 1110 & $1_{3-8}$ & 39 & East : & side & West & $\begin{array}{l}\text { Fallow, plowed, clods, some stubble. } \\
\text { Dry. }\end{array}$ \\
\hline$"$ & 1110 & $1_{3-9}$ & 39 & $"$ & $"$ & West & Same as $3-8$, closer view. \\
\hline$"$ & 1118 & $1_{3-10}$ & 39 & NW cos & rner & -- & $\begin{array}{l}\text { Sky condition, clear, hot, light breeze, } \\
\text { hazy dust. }\end{array}$ \\
\hline$"$ & 1124 & $1_{3-11}$ & 38 & West & side & $\mathrm{NE}$ & $\begin{array}{l}\text { Grapes. Evidence of irrigation but soil } \\
\text { dry at surface. }\end{array}$ \\
\hline$"$ & 1126 & $3-12$ & - & $\mathrm{NE} \mathrm{cos}$ & rner & SW & Pecan trees. \\
\hline$"$ & -- & $3-13$ & - & $\rightarrow$ & & East & Sign on west side of MAC and plane. \\
\hline$"$ & -- & $3-14$ & - & - & & East & Sign on west side of MAC. \\
\hline$"$ & -- & $3-15$ & -- & -- & & East & Sign by SE corner of field 33. \\
\hline 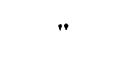 & 1133 & $1_{3-16}$ & 17 & South & side & North & Fallow, plowed, clods. Dry. \\
\hline$"$ & 1133 & $1_{3-17}$ & 17 & $"$ & $"$ & North & Same as $3-16$, closer view. \\
\hline$"$ & 1135 & $13-18$ & 15 & $"$ & $"$ & North & Cotton. Dry. \\
\hline$"$ & 1135 & $1_{3-19}$ & 15 & $"$ & $"$ & North & Same as $3-18$, closer view. \\
\hline$"$ & 1137 & $1_{3-20}$ & 13 & $"$ & $"$ & North & $\begin{array}{l}\text { Alfalfa. Sparser at west side. Soil } \\
\text { damp at surface. Humid. }\end{array}$ \\
\hline$"$ & 1137 & $1_{3-21}$ & 13 & $"$ & $"$ & North & Same as $3-20$, closer view. \\
\hline$"$ & 1143 & $13-22$ & 22 & $"$ & $"$ & North & Cotton. Dry. \\
\hline. & 1143 & $13-23$ & 22 & $"$ & $"$ & North & Same as $3-22$, closer view. \\
\hline$"$ & 1146 & $13-24$ & 21 & $"$ & $"$ & North & Cotton. Dry. \\
\hline$"$ & 1146 & $1_{3-25}$ & 21 & $"$ & $"$ & North & Same as $3-24$, closer view. \\
\hline$"$ & 1149 & $1_{3-26}$ & 20 & $"$ & $"$ & North & Cotton. Dry. \\
\hline
\end{tabular}


Table 2.--Photographs (35-millimeter slides) taken of fields on the demonstration farm. Maricopa Agricultural Center, and of scientists and equipment on September $28,1989--$ Cont inued

\begin{tabular}{|c|c|c|c|c|c|c|c|}
\hline Date & Time & Slide & $\begin{array}{l}\text { Field } \\
\text { number }\end{array}$ & Locat & ion & $\begin{array}{l}\text { View } \\
\text { direction }\end{array}$ & Description \\
\hline $9 / 28 / 89$ & 1149 & $1_{3-27}$ & 20 & South & side & North & Same as $3-26$, closer view. \\
\hline$"$ & 1151 & $1_{3-28}$ & 19 & $"$ & $"$ & North & Cotton. Dry. \\
\hline$"$ & 1151 & $13-29$ & 19 & $"$ & $"$ & North & Same as $3-28$, closer view. \\
\hline$"$ & -- & $1_{3-30}$ & 11 & North & side & South & Alfalfa. Soil darmp. \\
\hline$"$ & -- & $1_{3-31}$ & 11 & $"$ & $"$ & South & Same as $3-30$, closer view. \\
\hline$"$ & 1156 & $3-32$ & 19 & East $s$ & ide & SW & Well. \\
\hline$"$ & 1200 & $13-33$ & 23 & $"$ & $"$ & West & Cotton. Dry. \\
\hline$"$ & 1202 & $3-34$ & 22 & West $s$ & ide & East & Cotton. Dry. \\
\hline$"$ & -- & $13-35$ & 23 & East $s$ & side & West & Cotton. Dry. \\
\hline$"$ & 1207 & $1 / 3-36$ & 25 & North & side & South & $\begin{array}{l}\text { Fallow, plowed, clods, some rounded } \\
\text { clods. Dry. }\end{array}$ \\
\hline$"$ & 1207 & $13-37$ & 25 & $"$ & $"$ & South & Same as $3-36$, closer view. \\
\hline$"$ & -- & $14-1$ & 26 & East $s$ & side & West & Fallow at north end of field. Dry. \\
\hline$"$ & 1214 & $1_{4-2}$ & 26 & $"$ & $"$ & West & Cotton. Dry. \\
\hline$"$ & 1214 & $14-3$ & 26 & $"$ & $"$ & West & Same as $4-2$, closer view. \\
\hline$"$ & 1216 & $14-4$ & 25 & West $s$ & side & SE & Fallow with different surfaces. Dry. \\
\hline$"$ & 1225 & $4-5$ & 11 & Middle & & -- & $\begin{array}{l}\text { Weather station in alfalfa field. Soil } \\
\text { damp, humid. Flowers on alfalfa. }\end{array}$ \\
\hline$"$ & 1225 & $4-6$ & 11 & $"$ & & -- & Same as $4-5$, wider view. \\
\hline$"$ & -- & $4-7$ & 11 & $"$ & & -- & Alfalfa. \\
\hline$"$ & -- & $4-8$ & 11 & $"$ & & -- & Weather-station radiometer. \\
\hline$"$ & -- & 4-9 & 11 & $"$ & & North & Weather station in alfalfa field. \\
\hline$"$ & -- & $4-10$ & 11 & $"$ & & SE & Weather station in alfalfa field. \\
\hline$"$ & 1230 & $4-11$ & 11 & $"$ & & Close up & Alfalfa with flowers. \\
\hline " & -- & $1_{4-12}$ & -- & Plane & park & -- & $\begin{array}{l}\text { People packing equipment. Paul Pinter, } \\
\text { Ray Jackson, and Bob Anderson. }\end{array}$ \\
\hline " & - & $14-13$ & -- & $"$ & $"$ & -- & $\begin{array}{l}\text { People calibrating and packing } \\
\text { equipment. Tom Clarke, Stephanie } \\
\text { Johnson, Susan Moran, and Ray Jackson. }\end{array}$ \\
\hline$"$ & - & $14-14$ & -- & $"$ & $"$ & -- & Same as $4-13$ \\
\hline$"$ & -- & $1_{4-15}$ & -- & $"$ & $"$ & -- & $\begin{array}{l}\text { Same as 4-13. Tom Clarke, Stephanie } \\
\text { Johnson, and Susan Moran. }\end{array}$ \\
\hline
\end{tabular}


Table 2. - Photographs (35-millimeter slides) taken of fields on the demonstration farm, Maricopa Asricultural Center, and of scientists and equipment on September $28,1989--C o n t i n u e d$

\begin{tabular}{|c|c|c|c|c|c|c|}
\hline Date & Time & Slide & $\begin{array}{l}\text { Field } \\
\text { number }\end{array}$ & Location & $\begin{array}{c}\text { View } \\
\text { direction }\end{array}$ & Description \\
\hline $9 / 28 / 89$ & 1700 & $4-16$ & -- & Middle & SE & $\begin{array}{l}\text { Weather station in mowed (but not baled) } \\
\text { alfalfa field. Murphy Farm. }\end{array}$ \\
\hline$"$ & 1700 & $4-17$ & -- & Middle & SE & Same as $4-16$. \\
\hline$"$ & 1700 & $4-18$ & -- & $"$ & SE & Same as $4-16$. \\
\hline$"$ & 1700 & $4-19$ & -- & $"$ & ESE & Same as $4-16$ \\
\hline$"$ & 1757 & $4-20$ & -- & $"$ & $\mathrm{NE}$ & $\begin{array}{l}\text { Weather station in alfalfa field. } \\
\text { Ak-Chin Farm. }\end{array}$ \\
\hline$"$ & 1757 & $4-21$ & -- & $"$ & NE & Same as $4-20$. \\
\hline$"$ & 1757 & $4-22$ & - & $"$ & $\mathrm{NE}$ & Same as $4-20$ \\
\hline$"$ & 1757 & $4-23$ & -- & $"$ & $N E$ & Same as $4-20$ \\
\hline$"$ & 1757 & $4-24$ & -- & $"$ & NE & Same as $4-20$. \\
\hline$"$ & 1758 & $4-25$ & -- & West side & ENE & Same as $4-20$. \\
\hline
\end{tabular}

${ }^{1}$ Duplicate slides at the U.S. Water Conservation Laboratory, Phoenix, AZ.

One-mile wide fields are divided into 8 parts called benches. Benches differ in altitude and are numbered from east to west.

${ }^{3} \mathrm{Use}$ of brand names in this report is for identification only and does not constitute endorsement by the U.S. Geological Survey. 